\title{
HPLC-FLD methods to quantify chloroaluminum phthalocyanine in nanoparticles, plasma and tissue: application in pharmacokinetic and biodistribution studies
}

\author{
Líliam Teixeira Oliveira $^{\mathrm{a}}$, Giani Martins Garcia ${ }^{\mathrm{a}}$, Eunice Kazue Kano ${ }^{\mathrm{a}, 1}$, Antônio Cláudio Tedesco ${ }^{\mathrm{b}}$, \\ Vanessa Carla Furtado Mosqueira ${ }^{a, *}$
}

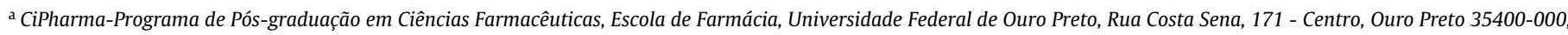
Minas Gerais, MG, Brazil

b Departamento de Química, FFCL-RP, Universidade de São Paulo, Av. Bandeirantes, 3900 - Monte Alegre, Ribeirão Preto 14040-901, São Paulo, SP, Brazil

\section{A R T I C L E I N F O}

\section{Article history:}

Received 22 December 2010

Received in revised form 12 April 2011

Accepted 16 April 2011

Available online 22 April 2011

\section{Keywords:}

Chloroaluminum phthalocyanine

High-performance liquid

chromatography-fluorescence detection

Nanocapsules

Photodynamic therapy

Fluorescent dye

\begin{abstract}
A B S T R A C T
Analytical and bioanalytical methods of high-performance liquid chromatography with fluorescence detection (HPLC-FLD) were developed and validated for the determination of chloroaluminum phthalocyanine in different formulations of polymeric nanocapsules, plasma and livers of mice. Plasma and homogenized liver samples were extracted with ethyl acetate, and zinc phthalocyanine was used as internal standard. The results indicated that the methods were linear and selective for all matrices studied. Analysis of accuracy and precision showed adequate values, with variations lower than $10 \%$ in biological samples and lower than $2 \%$ in analytical samples. The recoveries were as high as $96 \%$ and $99 \%$ in the plasma and livers, respectively. The quantification limit of the analytical method was $1.12 \mathrm{ng} / \mathrm{ml}$, and the limits of quantification of the bioanalytical method were $15 \mathrm{ng} / \mathrm{ml}$ and $75 \mathrm{ng} / \mathrm{g}$ for plasma and liver samples, respectively. The bioanalytical method developed was sensitive in the ranges of $15-100 \mathrm{ng} / \mathrm{ml}$ in plasma and $75-500 \mathrm{ng} / \mathrm{g}$ in liver samples and was applied to studies of biodistribution and pharmacokinetics of AlClPc.
\end{abstract}

(c) 2011 Elsevier B.V. All rights reserved.

\section{Introduction}

Phthalocyanine $(\mathrm{Pc})$ derivatives have attracted much attention because of their particular properties and have been used as photosensitizers and as fluorescent dyes. They have been investigated in several applicable materials, such as chemical sensors, liquid crystals and nonlinear optics. They have also been used in the pharmaceutical field as photosensitizers for photodynamic therapy (PDT) [1-4]. PDT is a bimodal therapeutic strategy based on a photosensitizer activated by visible light [2]. This technique has been proposed as an alternative or as a complement to conventional protocols in the treatment of malignant tumors [3]. Currently, PDT based on classical photosensitizers, hematoporphyrin or synthetic porphyrin derivatives is in preclinical and clinical use [5-8].

PDT based on phthalocyanines has remarkable medical potential because of improved selectivity and efficiency against tumor cells and microorganism proliferation $[9,10]$. Phthalocyanines exhibit a high absorption coefficient in the visible region

\footnotetext{
* Corresponding author. Tel.: +55 31 35591032; fax: +55 3135591628 .

E-mail addresses: vamosqueira@gmail.com, mosqueira@ef.ufop.br (V.C.F. Mosqueira).

1 Present address: Faculdade de Ciências Farmacêuticas da Universidade de São Paulo, Brazil.
}

of the spectrum, primarily in the phototherapeutic window $(600-800 \mathrm{~nm})$, and have a long lifetime of the triplet excited state in photosensitizers, which is necessary to produce ${ }^{1} \mathrm{O}_{2}$ (the toxic species) efficiently [11]. These features have led to proposals for their development for clinical use. However, some drawbacks related to phthalocyanine solubility and bioavailability must be addressed for it to be suitable for clinical use, particularly with chloroaluminum phthalocyanine $(\mathrm{AlClPc})$, which is very hydrophobic [12]. AlClPc presents optimal characteristics for use in PDT, such as long-lived excited singlet and triplet states [11]. Furthermore, despite the growing knowledge in the photophysical and photochemical aspects of AlClPc and in its medical applications, there is a slow evolution in the analytical methodologies for its determination in biological samples. The methods previously reported to determine AlClPc concentrations in biological samples are based on spectrophotometric and spectrofluorimetric assays [13,14]. Optical quantification methods in bulk tissues or homogenates are generally restricted in their detection limit and accuracy. These methods have not been validated in general for the AlClPc assay in biological matrices to provide the required selectivity, sensitivity, precision, accuracy, stability and recovery. Thus, a suitable liquid chromatographic method that will allow analysis of AlClPc in pre-clinical and clinical studies is still lacking.

Additionally, intravenous administration of the hydrophobic AlClPc is greatly hampered by its low solubility. Thus, this specific 

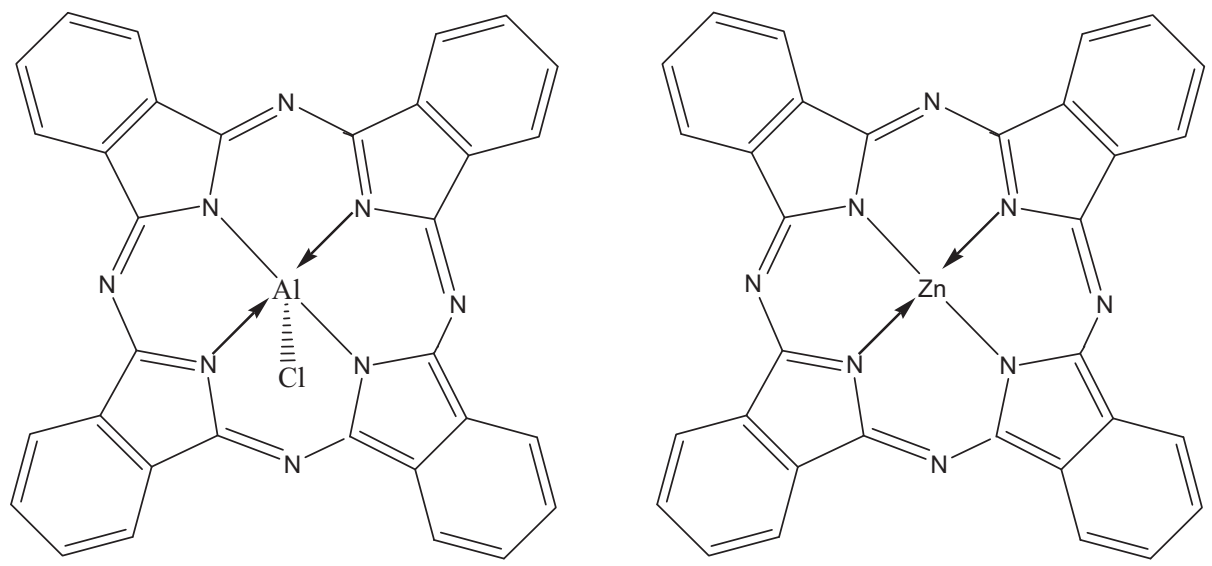

Fig. 1. Chemical structures of AlClPc and ZnPc.

physicochemical characteristic of AlClPc requires advanced technologies to formulate nanometric delivery systems, to improve its dispersion in water, enhance the specific uptake by targeted tissues and improve PDT efficiency $[15,16]$. Recently, Siqueira-Moura et al. described validated spectrophotometric and spectrofluorimetric methods for AlClPc quantification in nanocarriers in the ranges of $0.50-3.00$ and $0.05-1.00 \mu \mathrm{g} / \mathrm{ml}$, respectively [17]. However, AlClPc was not determined in biological samples and no extraction procedure was described. A literature survey indicated that there was no report of a validated method of high-performance liquid chromatography with fluorescence detection (HPLC-FLD) for the determination of AlClPc in biological samples and nanocarriers.

In this study, a suitable analytical method for the quantitative analysis of AlClPc in three different nanocapsule formulations as well as in biological samples of mice plasma and liver was developed and validated using HPLC-FLD. The tissue concentrations of $\mathrm{AlClPc}$ in the livers and plasma of mice $20 \mathrm{~min}$ after intravenous administration were measured. Furthermore, the pharmacokinetic profile of free AlClPc solution and AlClPc loaded in PLA-NC were determined to illustrate the application of the method described.

\section{Experimental}

\subsection{Chemicals}

AlClPc (chloro(29H,31H-phthalocyaninato)aluminum) and ZnPc (zinc(II)phthalocyanine) were purchased from Sigma (Sigma-Aldrich Co., St. Louis, MO, USA). The acetone, methanol, and dimethylformamide used for the HPLC analyses were HPLC grade and were purchased from Tedia (Brazil). Milli-Q water was purified using a Symplicity ${ }^{\circledR}$ System (Millipore, Bedford, USA) and was used to prepare all the solutions. Soy lecithin (Epikuron 170) was a gift from Lucas Meyer (France). The purified medium-chain triglyceride (MCT) was purchased from Hulls (Germany). Ethyl acetate, polyethylene glycol 300 and dimethyl sulfoxide (analytical grade) were purchased from Vetec (Rio de Janeiro, Brazil). PLA [poly(D,L-lactide), Mw 75,000-120,000 g/mol], low molecular weight chitosan (CS) and Poloxamer 188 were purchased from Sigma-Aldrich (Brazil). PLA-PEG [poly(D,L-lactide)-copolyethylenoglycol average $\mathrm{Mw} 66,000 \mathrm{~g} / \mathrm{mol}]$ with a PEG block of Mn $5000 \mathrm{~g} / \mathrm{mol}$ was a gift from Alkermes (Cambridge, USA) and was used without further purification.

\subsection{Nanocapsules preparation}

AlClPc loaded-nanocapsules, $0.1 \mathrm{mg} / \mathrm{ml}$ of PLA and PLA-PEG were prepared according to Fessi et al. [18] and Mosqueira et al. [19], respectively. Briefly, the polymer $(0.6 \%, w / v)$, oil $(0.75 \%, w / v)$, soy lecithin $(0.75 \%, \mathrm{w} / \mathrm{v})$ and AlClPc $(0.1 \mathrm{mg} / \mathrm{ml})$ were dissolved in acetone $(15 \mathrm{ml})$. The organic phase was poured into the aqueous phase $(30 \mathrm{ml})$ containing poloxamer $188(0.75 \%, \mathrm{w} / \mathrm{v})$, and the solution was stirred for $10 \mathrm{~min}$. The solvents were evaporated under reduced pressure up to $10 \mathrm{ml}$ (Heidolph Rotary Evaporator, Germany). PLA-CS nanocapsules (NC) were prepared by the same procedure above, replacing poloxamer by the chitosan $(0.25 \%, \mathrm{w} / \mathrm{v})$ in the aqueous phase. The size of the particles was determined by photon correlation spectroscopy (PCS) (Nanosizer N5 Plus Beckmann Counter, USA) after an appropriate dilution of the samples in ultra-pure Milli-Q water.

\subsection{Calibration standards}

A stock solution $(100 \mu \mathrm{g} / \mathrm{ml})$ of AlClPc was prepared in ethanol, and ZnPc was used as internal standard (I.S.) because of its solubility profile in ethanol, its chemical stability and chemical similarity to AlClPc (Fig. 1). Furthermore, it was previously used as an I.S. in quantitative assays in biological samples [20]. Stock solutions of AlClPc and ZnPc (I.S.) in ethanol at concentrations of $1 \mu \mathrm{g} / \mathrm{ml}$ and $5 \mu \mathrm{g} / \mathrm{ml}$, respectively, were prepared as standards and quality control samples (QC) for AlClPc quantification in biological matrices. Calibration curves were constructed using AlClPc concentrations of $150 \mathrm{ng} / \mathrm{ml}, 200 \mathrm{ng} / \mathrm{ml}, 400 \mathrm{ng} / \mathrm{ml}, 500 \mathrm{ng} / \mathrm{ml}, 800 \mathrm{ng} / \mathrm{ml}, 900 \mathrm{ng} / \mathrm{ml}$ and $1000 \mathrm{ng} / \mathrm{ml}$ (working solutions). The working solutions were prepared via the serial dilution method from the stock solution and all solutions were stored at $-20^{\circ} \mathrm{C}$.

\subsection{Chromatographic conditions}

The HPLC system employed consisted of a Waters Alliance 2695 separation module composed of an autosampler, pump, column oven and fluorescence detector (Waters 2475). The separation was performed on a $150 \mathrm{~mm} \times 4.6 \mathrm{~mm}, 4-\mu \mathrm{m}$ particle size C18 Gemini Phenomenex column protected by a Phenomemex security guard AJO-7597 C18 column $(2 \mathrm{~mm} \times 4.6 \mathrm{~mm}$, $3 \mu \mathrm{m})$. The isocratic mobile phase consisted of a mixture of methanol/acetone/dimethylformamide (90:5:15, v/v/v). It was prepared daily, degassed before use and pumped at $1 \mathrm{ml} / \mathrm{min}$. The analysis was performed at $30^{\circ} \mathrm{C}$. The injection volume was $30 \mu \mathrm{l}$ for biological samples and $10 \mu \mathrm{l}$ for the other samples. The column eluent was monitored with a fluorescence detector at wavelengths of $610 \mathrm{~nm}$ and $675 \mathrm{~nm}$, for excitation and emission, respectively.

\subsection{Analytical method}

The nanocapsules were analyzed by an analytical method, which was validated in accordance with International Conference on 
Harmonization [ICH] guidelines [21]. Calibration solutions were prepared by diluting the stock solution $(100 \mu \mathrm{g} / \mathrm{ml})$ in ethanol to obtain concentrations of $0.025,0.05,0.1,0.5,1.0,2.0,4.0$ and $5.0 \mu \mathrm{g} / \mathrm{ml}$. The calibration curve was constructed by linear regression of the plot of AlClPc peak area against the corresponding concentration. Quality-control samples were prepared at concentrations of $0.5,2.0$ and $4.0 \mu \mathrm{g} / \mathrm{ml} \mathrm{AlClPc}$ to determinate the precision and accuracy of the analytical method. The selectivity in the presence of polymers and excipients used in the NC formulations was investigated by preparing a mobile phase spiked with drug-free PLA, PLA-PEG and PLA-CS nanocapsules. The nanocapsule formulations were completely disrupted and dissolved in the mobile phase before the chromatographic analysis.

The quantification of AlClPc in the polymeric nanocapsules of PLA, PLA-PEG and PLA-CS was performed as described below. The percentage of encapsulation (\% drug loading) of AlClPc in nanocapsules was calculated as the difference between the total drug in the final colloidal suspension and the free drug in the external aqueous phase. The AlClPc not encapsulated can be found dissolved in the external aqueous phase or precipitated. To determine the amount of drug that precipitated in the colloidal NC suspension (not encapsulated), the solution was filtered using a $0.8 \mu \mathrm{m}$ filter to remove AlClPc crystals. The fraction of AlClPc dissolved in the external phase (ultrafiltrate) was assessed by centrifuging $400 \mu$ l samples of NC (after filtration) in an AMICOM device (Microcon Ultrafilter, MWCO 50,000 Da, Millipore ${ }^{\circledR}$ ) at $500 \times g$ for $15 \mathrm{~min}$. The AlClPc associated with the NC was retained in the upper compartment of the device.

The amount of AlClPc loaded on the NC was determined from Eq. (1) and the encapsulation efficiency of AlClPc into the NC was determined by Eq. (2), where $A=$ concentration:

$(\%)=\frac{A^{\text {total }}-A^{\text {ultrafiltrate }}}{A^{\text {total }}} \times 100$

$(\%)=\frac{A^{\text {total }}-A^{\text {ultrafiltrate }}}{A^{\text {weighed }}} \times 100$

For total AlCIPc determination in the NC, $100 \mu$ l of the NC suspension was added to $2.5 \mathrm{ml}$ of acetonitrile, vortexed for $5 \mathrm{~min}$ (Vortex Instrument, IKA, Germany), and centrifuged; $25 \mu \mathrm{l}$ of the supernatant was then injected into the HPLC system. This step disrupted the nanocapsules and released the drug. For quantification of the unencapsulated drug, $50 \mu$ l of the ultrafiltrate was mixed with $200 \mu$ l acetonitrile. The mixture was centrifuged and the supernatant assayed using the HPLC. All samples analyzed by HPLC were filtered using a $0.45 \mu \mathrm{m}$ filter $(4 \mathrm{~mm})$ before injection $(25 \mu \mathrm{l})$ into the HPLC system. The analysis was performed in triplicate.

\subsection{Animals}

The in vivo experiments were approved by the Ethics Committee on Animal Experimentation of the Universidade Federal de Ouro Preto, Brazil (protocol no. 2009/29) and are in compliance with the Guide for the Care and Use of Laboratory Animals recommended by the Institute of Laboratory Animals Resources [22]. Female Swiss mice were used in this study. They weighed 23.5-24.5 $\mathrm{g}$ and were maintained at the following environmental conditions: $12 \mathrm{~h}$ day/night cycle, temperature $22 \pm 2{ }^{\circ} \mathrm{C}$, standard diet and water ad libitum.

\subsection{Biological sample preparation}

Plasma and liver samples from drug-free animals were used as blanks for method validation. Blood from anesthetized mice (ketamine $60 \mathrm{mg} / \mathrm{kg}$ and xylazine $7.5 \mathrm{mg} / \mathrm{kg}$ ) was collected in
Eppendorf tubes containing heparin. The plasma was immediately separated by centrifugation at $400 \times g$ for $10 \mathrm{~min}$ and was then aliquoted and stored at $-80^{\circ} \mathrm{C}$ until analyzed. After animals were euthanized, the tissues were removed, washed with cold saline and blotted onto filter paper. Pieces of liver $(200 \mathrm{mg})$ were cut, weighed and stored at $-80^{\circ} \mathrm{C}$ until needed.

For the quantification of AlClPc in plasma, plasma samples $(80 \mu \mathrm{l})$ were placed into Eppendorf microcentrifuge tubes; AlClPc standards $(10 \mu \mathrm{l})$ of each working solution and $10 \mu \mathrm{l}$ of I.S. (ZnPc $5 \mu \mathrm{g} / \mathrm{ml}$ ) were added to each tube. The solutions were mixed in a vortex and extracted with $1 \mathrm{ml}$ of ethyl acetate. Then, the samples were shaken on a vortex for $10 \mathrm{~min}$ at the same speed for all samples. Afterwards, tubes were centrifuged at $9300 \times \mathrm{g}$ for $10 \mathrm{~min}$ and the resulting upper organic layers were pooled. For each sample, this procedure was repeated 3 times and the organic layers were pooled, filtered using $0.45-\mu \mathrm{m}$ filters (Millipore, USA) and evaporated to dryness in a nitrogen-stream. After the extraction procedure, each sample was reconstituted in $100 \mu \mathrm{l}$ of the mobile phase.

For quantification in the liver samples, thawed pieces liver ( $200 \mathrm{mg}$ ) were homogenized for $1 \mathrm{~min}$ in a round-bottom plastic tube with $1 \mathrm{ml}$ sodium phosphate buffer ( $\mathrm{pH}$ 6.5) at room temperature in an ultrasonic processor (Vibra cell ${ }^{\mathrm{TM}}$ VC750) with a metal rod titanium probe at $300 \mathrm{~W}$. The tissue homogenates were stored at $-80^{\circ} \mathrm{C}$ until the extraction. AlClPc was extracted from $80 \mu \mathrm{l}$ of the homogenate tissue using the same procedure described above for plasma samples.

\subsection{Assay performance}

The method was validated according to FDA guidelines for validation of bioanalytical methods [23]. The assay performance was evaluated through the determination of selectivity, limits of detection (LOD), limits of quantification (LOQ), the linearity over the tested concentration range (from 15 to $100 \mathrm{ng} / \mathrm{ml}$ and 75 to $500 \mathrm{ng} / \mathrm{g}$ for mice plasma and liver samples, respectively), recovery, accuracy, precision, and the stability of the bioanalytical method. Because of the light-absorbing properties of AlClPc, all samples were protected from light; preparations and extractions were performed in darkened rooms.

\subsubsection{Selectivity}

The selectivity of a bioanalytical method is generally defined as the lack of interfering peaks at the retention times of the assayed drug and the internal standard in the chromatograms [23]. The selectivity of the assay was investigated by processing and analyzing blanks prepared from six independent lots of plasma and liver samples controls.

\subsubsection{Limit of detection and limit of quantification}

The limit of detection (LOD) of the bioanalytical methods was defined as the lowest concentration level giving a peak area of three times the noise. The LOQ was determined as the lowest concentration on the standard calibration curve, which can be quantitatively determined with a precision of $20 \%$ and accuracy of $80-120 \%$ [23].

\subsubsection{Linearity}

The linearity of an analytical procedure is its ability (within a given range) to obtain test results that are directly proportional to the concentration (amount) of analyte in the sample [21]. The linearity of the bioanalytical assay for the test compounds was evaluated with a total of seven calibrations standards over the concentration range of $15-100 \mathrm{ng} / \mathrm{ml}$ for plasma and 75-500 ng/g for liver. Calibration curves were constructed by the linear least-squares regression analysis by plotting the peak-area ratios (AlClPc/I.S.) versus the drug concentrations. The calibration 
model was accepted if (a) the coefficient of correlation was greater than or equal to 0.98 and/or (b) residuals were within $\pm 20 \%$ at the lower limit of quantification and within $\pm 15 \%$ at all other calibration levels [23]. Five calibration curves were prepared from a blank sample (matrices samples processed with the internal standard), including the LOQ.

\subsubsection{Recovery}

The trueness of an analytical procedure refers to the closeness of agreement between a conventionally accepted value and a mean experimental value. The trueness was expressed as the percentage recovery of the target value assessed by the validation standards in the plasma and liver at three independent concentration levels: low, medium and high [23]. Recovery was calculated by comparing the mean peak area obtained from an extracted sample (recovered $\%)$ to the peak area obtained after the direct injection of a solution of the same drug concentration diluted in the mobile phase (100\%). The \% recovery was determined by comparing the concentrations of three quality control samples ( $n=6,20,40$ and $80 \mathrm{ng} / \mathrm{ml}$ for plasma samples and 100, 200 and $400 \mathrm{ng} / \mathrm{g}$ for liver samples) with unextracted reference standards containing the same amount of the analyte. The samples were prepared from blank plasma, which was extracted three times using ethyl acetate, vortexed for $10 \mathrm{~min}$ and centrifuged for $10 \mathrm{~min}$ at $9300 \times \mathrm{g}$. The supernatants were drawn and the standard solutions and internal standard solutions were added to provide the reference standards that were dried and reconstituted with the mobile phase for analysis.

\subsubsection{Accuracy and precision}

The intra-batch accuracy and precision were determined by the analysis of six replicates of low $(20 \mathrm{ng} / \mathrm{ml})$, medium $(40 \mathrm{ng} / \mathrm{ml})$ and high $(80 \mathrm{ng} / \mathrm{ml})$ concentrations of the analyte in mice plasma and low (100 ng/g), medium (200 ng/g) and high (400 ng/g) concentrations in mice liver. Inter-batch accuracy and precision were determined by the analysis of these quality control (QC) samples on three separate occasions. The overall precision of the method was expressed as relative standard deviation (RSD) and the accuracy of the method was expressed in terms of relative error. The accuracy and precision determined at each concentration level of the curve points must be within $\pm 15 \%$ of the respective nominal value.

\subsubsection{Stability}

The drug stability in biological matrices is a function of the storage conditions, the chemical properties of the drug and the matrix [23]. For all stability studies, low, medium and high plasma quality control (QC) samples were used in triplicate. The stability at $24 \mathrm{~h}$ (after the plasma sample extracts had been exposed to the same temperature as the autosampler environment) was determined for the analytes and for the I.S. The freeze-thaw stability of the samples was obtained over three freeze-thaw cycles, by thawing the QC samples unassisted at room temperature and then refreezing for $12-24 \mathrm{~h}$ followed by analysis. The bench-top stability was evaluated by keeping the QC samples at room temperature for $4 \mathrm{~h}$ and then the samples were processed and analyzed. The AlClPc stability was evaluated at $-80^{\circ} \mathrm{C}$ for 30 days. The concentration of AICIPC after each test condition was compared to the initial concentration added to the sample. The working solutions of AlClPc $(40 \mathrm{ng} / \mathrm{ml})$ and the I.S. ( $500 \mathrm{ng} / \mathrm{ml}$ ) were analyzed immediately after preparation and after being stored for 30 days at $-20^{\circ} \mathrm{C}$. Subsequently, the concentrations $(20,40$ and $80 \mathrm{ng} / \mathrm{ml}$ in plasma samples and 100 , 200 and $400 \mathrm{ng} / \mathrm{g}$ in liver samples) were compared.

\subsection{Application of the method}

To test the validated methods with real samples, experiments were undertaken to determine AlClPc in three types of nanocapsules of different chemical constitutions using the analytical method developed. The bioanalytical method was used to determine AlClPc concentrations in plasma and liver samples of healthy mice $(n=4)$. The mice received single intravenous doses of $50,100,200$ or $300 \mu l$ of $0.1 \mathrm{mg} / \mathrm{ml}$ AlClPc-loaded, PLA nanocapsules. The blood samples (approximately $500 \mu \mathrm{l}$ ) were collected in heparinized tubes $20 \mathrm{~min}$ after administration. Samples were immediately centrifuged for $10 \mathrm{~min}$ at $400 \times \mathrm{g}$, and the plasma was then frozen at $-80^{\circ} \mathrm{C}$ until analysis. After euthanasia of the mice, the livers were excised, washed with cold saline and blotted with filter paper. The quantification of AlClPc in biological samples was undertaken within $12-24 \mathrm{~h}$ post-collection of samples using the method described in Section 2.7. The QC samples were incorporated into the series on the same day of the analysis.

The pharmacokinetic study was performed with free AlClPc and AlClPc loaded in PLA-NC. The free AlClPc solution was prepared dissolving $400 \mu \mathrm{g}$ of the drug in $400 \mu \mathrm{l}$ of DMSO and $600 \mu \mathrm{l}$ of PEG 300. Afterward this solution was diluted in $5 \%$ glucose solution up to $0.1 \mathrm{mg} / \mathrm{ml}$ of AlClPc final concentration. The AlClPc solution and AlClPc PLA-NC suspension were filtered on a $0.8 \mu \mathrm{m}$ sterile filter and $150 \mu \mathrm{l}$ were administered intravenously in mice. At the indicated time intervals, 5, 20, $60 \mathrm{~min}$ and 3, 6, 12, 24, $48 \mathrm{~h}$ post-injection, blood $(200 \mu \mathrm{l})$ was collected in heparinized tubes, centrifuged for $15 \mathrm{~min}$ at $800 \times \mathrm{g}$ and plasma was collected. At $50 \mu \mathrm{l}$ of plasma samples $10 \mu \mathrm{l}$ of I.S. solution were added. The samples were then extracted by the method described above. To determine the amount of total fluorescent dye (AlClPc) in plasma, a total plasma volume per mouse of $4.9 \%$ of body weight was assumed [24]. The results were expressed as a percentage of the injected dose and are the mean \pm standard errors ( $n=6$ mice). To compare the different formulations, the area under the curve (AUC) (concentration $\times$ time) of free AlClPc and AlClPc loaded in PLA-NC were calculated by the trapezoidal method during the experimental period $\left(\mathrm{AUC}_{[0-48]}\right)$. The extrapolated area $\left(\mathrm{AUC}_{[t-\omega]}\right)$ was calculated by dividing the last experimental concentration $\left(C_{t}\right)$ by the rate constant of elimination $(K)$ because this area represented approximately $1 \%$ of the experimental AUC, it was neglected in our calculations. The statistical analysis was performed using Prisma ${ }^{\circledR}$ 5.0 software. The differences lower than $p<0.01$ were considered significant. Thus, the overestimated plasma clearance $(\mathrm{Cl})$ was calculated from the following equation: $\mathrm{Cl}=$ Dose $_{\mathrm{iv}} / \mathrm{AUC}_{0-48}$.

\section{Results and discussion}

Using the HPLC-FLD analytical method developed, AlClPc in nanocapsules exhibited a retention time of $1.97 \mathrm{~min}$ in the experimental conditions utilized and the calibration plot was linear in the concentration range investigated $(0.025-5.0 \mu \mathrm{g} / \mathrm{ml})$. The slopes, intercepts and the coefficients of determination were found to be $13979031(a), 356534(b)$ and $0.9997\left(r^{2}\right)$, respectively. The limits of detection (LOD) were calculated by the equation $\mathrm{LOD}=3.3 \sigma / \mathrm{S}$ and the limit of quantification (LOQ) was calculated by the equation $\mathrm{LOQ}=10 \sigma / \mathrm{S}$, where $\sigma$ is the standard deviation of $y$-intercepts of regression lines and $S$ is the slope of the calibration curve. The LOD and LOQ values were $1.12 \mathrm{ng} / \mathrm{ml}$ and $3.39 \mathrm{ng} / \mathrm{ml}$, respectively. The HPLC-FLD method showed more sensitivity than the spectrofluorimetric methods described by Siqueira-Moura et al. which showed LOD and LOQ values of 10 and $40 \mathrm{ng} / \mathrm{ml}$, respectively [17]. The chromatogram of AlClPc was not altered in the presence of polymers and other chemical constituents used in PLA, PLA-PEG and PLA-CS NC formulations, such as polymers, surfactants and oils in accordance with previously observed data (Fig. 2) [17]. To determine intra-assay accuracy and precision, six replicate analyses were performed at each of the three concentrations $(0.5,2.0$ and $4.0 \mu \mathrm{g} / \mathrm{ml})$. The means for quantification of AlClPc with quality control sam- 
Table 1

Accuracy and precision determined by analytical method.

\begin{tabular}{llllll}
\hline \multirow{2}{*}{$\begin{array}{l}\text { Concentration } \\
(\mu \mathrm{g} / \mathrm{ml})\end{array}$} & \multicolumn{2}{l}{ Precision (RSD \%) } & & \multicolumn{2}{l}{ Accuracy (\%) } \\
\cline { 2 - 3 } \cline { 5 - 6 } & Intra-day $^{\mathrm{a}}$ & Inter-day $^{\mathrm{a}}$ & & Intra-day $^{\mathrm{a}}$ & Inter-day $^{\mathrm{a}}$ \\
\hline 0.5 & 1.00 & 1.35 & & 101.43 & 101.84 \\
2.0 & 1.80 & 1.67 & & 99.77 & 101.88 \\
4.0 & 0.50 & 1.48 & & 101.90 & 100.59 \\
\hline
\end{tabular}

RSD, relative standard deviation

a $n=6$.

ples in the nanocapsules are summarized in Table 1 . The mean intra-day precision values for all samples were found to be within $0.50-1.80 \%$, and the inter-day precision values were between $1.35 \%$ and $1.67 \%$. The intra-day accuracy was found to range from 99.77 to $101.90 \%$ and 100.59 to $101.88 \%$ for the inter-day accuracy. Moreover, the relative standard deviation (RSD) obtained within-day and between-day experiments were below $2 \%$ in each case.

During the development and validation of the bioanalytical method of quantification for AlClPc in mice plasma and liver, five sets of calibration standards were prepared for each matrix. The retention times for AlClPc and for the I.S. at this point of study were 2.036 and $4.816 \mathrm{~min}$, respectively (Fig. 2). The assays were linear between $15 \mathrm{ng} / \mathrm{ml}$ and $100 \mathrm{ng} / \mathrm{ml}$ in plasma and between $75 \mathrm{ng} / \mathrm{g}$ and $500 \mathrm{ng} / \mathrm{g}$ in liver samples. The mean regression equation of five standard curves was: $y=(0.0235) x+(0.0207)$ for the plasma and $y=(0.0221) x+(0.1650)$ for the liver samples, where $y$ is the peak area ratio of AlClPc/ I.S. and $x$ is the concentration of AlCIPc. The calibration curves generated showed a good relationship between the area of the peak of AlClPc /I.S. $(y)$ and the respective AlClPc concentration $(x)$, with $r^{2}=0.9873$ for the plasma and $r^{2}=0.9913$ for the liver samples. The LOQ and LOD were found to be $15 \mathrm{ng} / \mathrm{ml}$ and $5 \mathrm{ng} / \mathrm{ml}$ and $75 \mathrm{ng} / \mathrm{g}$ and $25 \mathrm{ng} / \mathrm{g}$, respectively for plasma and liver samples. The limit of quantification in the liver samples ( $\mathrm{LOQ}=75 \mathrm{ng} / \mathrm{g}$ ) of the method developed herein was found to be better than those previously reported ( $\mathrm{LOQ}=100 \mathrm{ng} / \mathrm{g}$ ) [24].

The selectivity of the method was evaluated by processing drug-free plasma and liver samples obtained from six mice (Swiss, female) in a similar manner to the calibration standards and unknowns. Chromatograms were compared to determine if there was any interference from the matrix or from any of the assay reagents. The method was selective in both matrices. There were no endogenous interferences in plasma and liver from the mice in the determination of the AlClPc and the I.S. (Fig. 2). To quantify the AlClPc extracted from biological matrices, the inherent high fluorescence quantum yields of the Pcs were exploited. At this spectral range (600-700 nm), the intrinsic biomolecules, such as NADH, porphyrins and collagen displayed minimal excitability, hence had low auto fluorescence, which resulted in low interference. In the method developed here, good chromatographic resolution was achieved between the analyte (AlClPc) and the I.S. ( $\mathrm{ZnPc}$ ).

The precision of an analytical procedure expresses a close agreement between a series of measurements obtained from multiple sampling of the same homogeneous sample, whereas the accuracy of an analytical method describes a close test results obtained by the method to the true (nominal) value of the analyte. For plasma samples, intra-day precision and accuracy ranged from $0.99 \%$ to $5.50 \%$ and $98.27 \%$ to $107.31 \%$, respectively. For liver samples, intra-day precision and accuracy ranged from $10.06 \%$ to $12.71 \%$ and $90.95 \%$ to $103.15 \%$, respectively. Inter-day precision and accuracy ranged from $6.24 \%$ to $9.40 \%$ and $93.81 \%$ to $104.02 \%$ respectively for plasma samples and ranged from $11.44 \%$ to $13.87 \%$ and $99.48 \%$ to $104.48 \%$, respectively for the liver samples (Table 2). These results were within the limits established by FDA guidelines for the validation of bioanalytical methods [23].

The recovery values obtained for each matrix are presented in Table 3. The recovery was very efficient for the standard and the I.S. in plasma and liver samples. The mean percentages recovered were $96.11 \%$ and $99.27 \%$ for AlClPc and $96.68 \%$ and $95.76 \%$ for the I.S. in plasma and liver samples, respectively. The extraction proce-
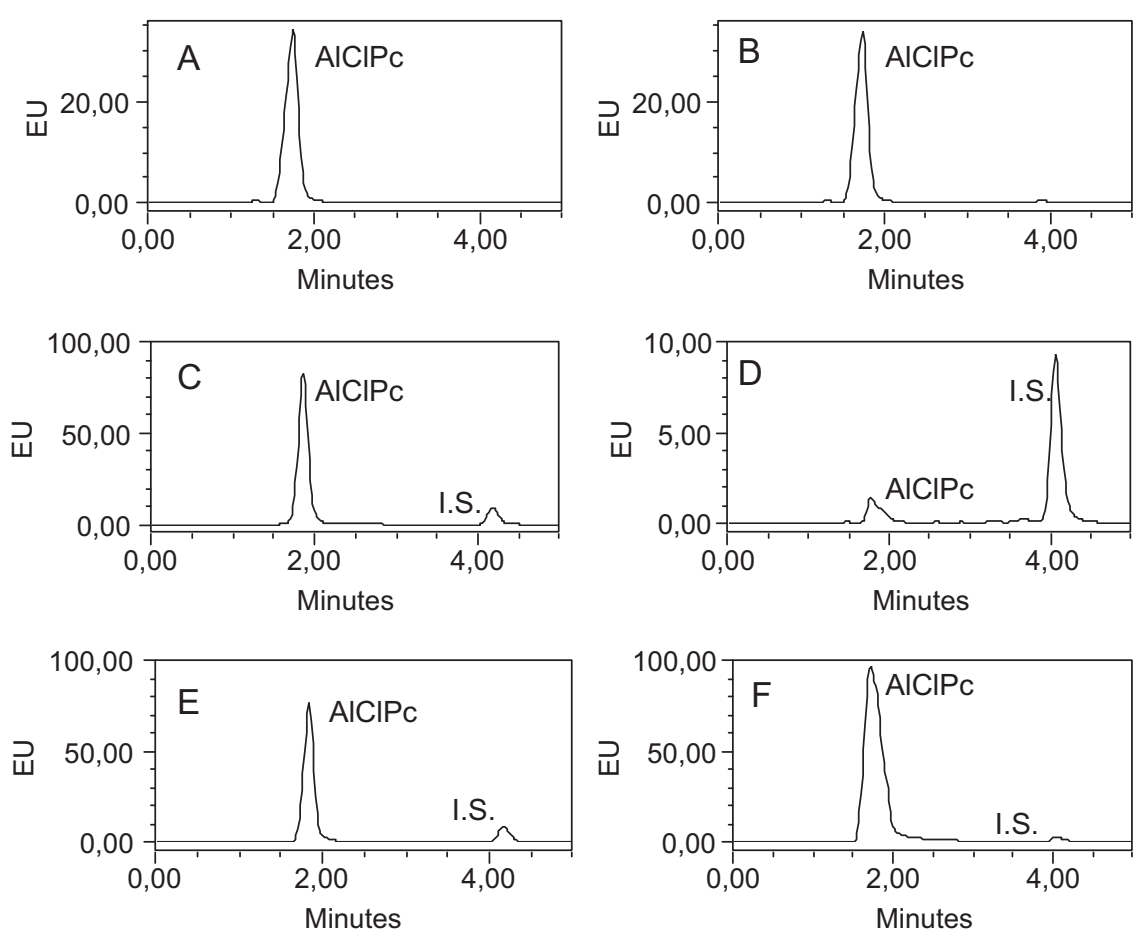

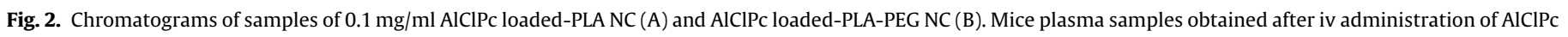
loaded PLA NC, $5 \mathrm{~min}$ (C) and $12 \mathrm{~h}$ (D). Mice liver samples obtained $20 \mathrm{~min}$ after administration of $50 \mu \mathrm{l}$ (E) and $100 \mu \mathrm{l}$ (F) of $0.1 \mathrm{mg} / \mathrm{ml}$ AlClPc PLA NC. 
Table 2

Intra- and inter-day precision and accuracy of AlCIPc determined by bioanalytical method.

\begin{tabular}{|c|c|c|c|c|c|}
\hline & \multirow[t]{2}{*}{ Concentration } & \multicolumn{2}{|c|}{ Precision (RSD \% $)^{\mathrm{a}}$} & \multicolumn{2}{|c|}{ Accuracy (\%) } \\
\hline & & Intra-day & Inter-day & Intra-day & Inter-day \\
\hline \multirow{3}{*}{ Plasma (ng/ml) } & 20 & 5.50 & 8.23 & 107.31 & 96.14 \\
\hline & 40 & 8.77 & 9.40 & 98.27 & 93.81 \\
\hline & 80 & 0.99 & 6.24 & 101.32 & 104.02 \\
\hline \multirow{3}{*}{ Liver (ng/g) } & 100 & 12.71 & 11.52 & 90.95 & 99.48 \\
\hline & 200 & 12.44 & 13.87 & 99.95 & 104.48 \\
\hline & 400 & 10.06 & 11.44 & 103.15 & 103.45 \\
\hline
\end{tabular}

RSD, relative standard deviation.

$n=6$

dure was the same for the liver and plasma samples and exhibited high percentages of extraction of AlCIPc from the samples. The extraction methodology was simple, fast and cost-effective because only one solvent was needed in low volumes and there was no need for further purification. Furthermore, one important advantage of this bioanalytical technique was that only $100 \mu$ l of plasma or $200 \mathrm{mg}$ of tissue were sufficient for obtaining results in a wide range of concentrations applicable to biodistribution, toxicology and pharmacokinetics studies, which would require small laboratory animals or samples, such as biopsies [14]. The ZnPc was found to be a suitable internal standard because of its chemical stability $[12,20]$, its similarity to AlClPc's structure and is the fact that it is easily recovered from plasma and liver samples (>95\%), as shown in Table 3.

Table 4 lists data for stability tests. Three sets of plasma samples at low, medium and high concentrations of QC were analyzed in all stability tests, and the results indicated that the AlCIPC was stable. Processed plasma and liver samples were stable for at least $24 \mathrm{~h}$ at room temperature in the autosampler. The samples were stable after the three freeze-thaw cycles. These results suggested that plasma and liver samples could be thawed and refrozen without compromising the integrity and accuracy of the samples. The working solutions of AlCIPc and I.S. were stable for 30 days at $-20^{\circ} \mathrm{C}$. The bench-top stability was investigated to ensure that AlClPc remained stable in plasma and liver samples at room temperature for $4 \mathrm{~h}$. The AlClPc concentrations $(n=5)$ found in plasma and tissue frozen at $-80^{\circ} \mathrm{C}$ were not statistically different $(p>0.05)$ from the concentration measured at the beginning of study. This ensemble of results confirmed AlClPc's long-term stability and its suitability as a fluorescent dye in NC formulations and in biological samples. The results obtained on the stability studies indicated that samples can be stored at $-80^{\circ} \mathrm{C}$ for thirty days without loss of the analyte. The fluorescence response after contact of AlClPc with

Table 3

Absolute recovery of AlCIPc and ZnPc from plasma and liver matrices.

\begin{tabular}{llcc}
\hline Concentration $^{\mathrm{a}}$ & \multicolumn{2}{c}{ Recovery (\%) } \\
\cline { 3 - 4 } & & AlClPc & ZnPc (I.S.) \\
\hline \multirow{3}{*}{ Plasma (ng/ml) } & 20 & 94.10 & 96.58 \\
& 40 & 95.67 & 95.33 \\
& 80 & 98.56 & 98.13 \\
& Mean & 96.11 & 96.68 \\
RSD & 1.92 & 1.18 \\
& & & 89.96 \\
Liver $(\mathrm{ng} / \mathrm{g})$ & 100 & 96.32 & 96.34 \\
& 200 & 101.53 & 100.97 \\
& 400 & 99.96 & 95.76 \\
& Mean & 99.27 & 5.77 \\
\hline
\end{tabular}

$n=5$.

b ZnPc is internal standard.

c Relative standard deviation. solvent was time-independent because of the solvent used in the method; in comparison, in a previously reported method, the contact time between the biological sample and solvent affected the fluorescence response [14].

Direct chromatographic analysis of the majority of the phthalocyanine derivatives was difficult because of the poor solubility of these compounds in the mobile phase, except for the mobile phases containing concentrated $\mathrm{H}_{2} \mathrm{SO}_{4}$ and dimethyl sulfoxide [26]. Phthalocyanine derivatives were problematic HPLC analytes because of the presence of an extended $\pi$ region that is prone to adsorption and $\pi$-stacking phenomena. Their chromatographic separation was possible under the conditions of normal-phase and reversed-phase HPLC because of polar and nonpolar groups on the molecule $[25,26]$. Dimethylformamide was mixed with methanol and acetone to constitute the eluent phase. It was successful in solubilizing the AlCIPc and reducing the adsorption on the stationary phase, probably by improving interaction between the analyte and solvents.

\subsection{Application}

Nanocapsules with different superficial characteristics were prepared by the nanoprecipitation method $[18,19]$ with mean sizes of between 155 and $256 \mathrm{~nm}$. The AlClPc content in PLA, PLA-PEG and PLA-CS NC was determined using the validated analytical method described herein.

The precipitation of AlClPc was sometimes observed after preparation of the NC; thus, the colloidal suspensions were filtered using a $0.8 \mu \mathrm{m}$ membrane before the AlClPc determination in each nanocapsule formulation. The AlClPc payload in the nanocapsules ranged from $35.3 \%$ to $86.4 \%$ (Table 5 ), as determined previously for other drugs [19]. The higher the AlCIPc precipitation in the formulation, the lower the efficiency of the encapsulation process. It was influenced by the NC coating composition. Chitosan reduces AlClPc association with NC. However, drug loading was not influenced by the composition of the external surface of nanocapsules at $0.1 \mathrm{mg} / \mathrm{ml}$, indicating that AlClPc was probably entrapped in the oily core.

A recently published study reported the validated spectrophotometric and spectrofluorimetric methods for AlClPc quantification in nanocarriers [17]. However, the method described herein also allowed the quantification of the AlClPc with the same sensitivity and with better selectivity in biological samples. The bioanalytical method applied herein was able to determine AlClPc concentrations in plasma and liver samples $20 \mathrm{~min}$ after PLA NC was intravenously administered in mice. A biodistribution study was used to investigate the effect of increasing doses of particles on the mice plasma and livers concentrations of AlClPc (Fig. 3). The doses of nanoparticles administered to the animals were particularly high to saturate particle uptake by the mononuclear phagocytic system (MPS). It was necessary to dilute the samples of plasma from treated mice 5-10 fold with blank mice plasma before the extrac- 
Table 4

Stability data of AlClPc in plasma and liver samples.

\begin{tabular}{|c|c|c|c|c|}
\hline Stability & $\begin{array}{l}\text { Nominal } \\
\text { concentration }\end{array}$ & $\begin{array}{l}\text { Measured } \\
\text { concentration } \\
(\mathrm{ng} / \mathrm{ml})\end{array}$ & $\begin{array}{l}\text { Precision } \\
\text { (RSDa\%) }\end{array}$ & Accuracy (\%) \\
\hline \multicolumn{5}{|l|}{ Plasma } \\
\hline \multirow{3}{*}{ Bench top $4 \mathrm{~h}$} & $20 \mathrm{ng} / \mathrm{ml}$ & 20.95 & 7.05 & 104.27 \\
\hline & $40 \mathrm{ng} / \mathrm{ml}$ & 20.44 & 0.97 & 101.09 \\
\hline & $80 \mathrm{ng} / \mathrm{ml}$ & 75.51 & 2.42 & 94.38 \\
\hline \multirow{3}{*}{ Autosampler $24 \mathrm{~h}$} & $20 \mathrm{ng} / \mathrm{ml}$ & 20.93 & 5.08 & 103.23 \\
\hline & $40 \mathrm{ng} / \mathrm{ml}$ & 43.22 & 7.46 & 108.05 \\
\hline & $80 \mathrm{ng} / \mathrm{ml}$ & 81.46 & 7.94 & 101.83 \\
\hline \multirow{3}{*}{ Freeze-thaw three cycles } & $20 \mathrm{ng} / \mathrm{ml}$ & 18.40 & 2.32 & 92.02 \\
\hline & $40 \mathrm{ng} / \mathrm{ml}$ & 38.36 & 2.36 & 95.89 \\
\hline & $80 \mathrm{ng} / \mathrm{ml}$ & 81.45 & 12.53 & 101.82 \\
\hline \multicolumn{5}{|l|}{ Liver } \\
\hline \multirow{3}{*}{ Bench top $4 \mathrm{~h}$} & $100 \mathrm{ng} / \mathrm{g}$ & 101.15 & 1.55 & 101.15 \\
\hline & $200 \mathrm{ng} / \mathrm{g}$ & 198.76 & 6.30 & 99.38 \\
\hline & $400 \mathrm{ng} / \mathrm{g}$ & 402.64 & 8.25 & 100.66 \\
\hline \multirow{3}{*}{ Autosampler $24 \mathrm{~h}$} & $100 \mathrm{ng} / \mathrm{g}$ & 100.75 & 8.10 & 100.75 \\
\hline & $200 \mathrm{ng} / \mathrm{g}$ & 209.05 & 9.48 & 104.52 \\
\hline & $400 \mathrm{ng} / \mathrm{g}$ & 428.8 & 9.91 & 107.20 \\
\hline \multirow{3}{*}{ Freeze-thaw three cycles } & $100 \mathrm{ng} / \mathrm{g}$ & 104.95 & 9.31 & 104.95 \\
\hline & $200 \mathrm{ng} / \mathrm{g}$ & 211.15 & 6.34 & 105.59 \\
\hline & $400 \mathrm{ng} / \mathrm{g}$ & 408.55 & 0.05 & 102.14 \\
\hline
\end{tabular}

a Relative standard deviation.

tion to fit the AlCIPc concentration in the biological samples within the calibration curves. The same technique was used for the liver samples. Fig. 3 shows that AlClPc concentrations in the plasma and liver samples are very high compared with the administered doses,

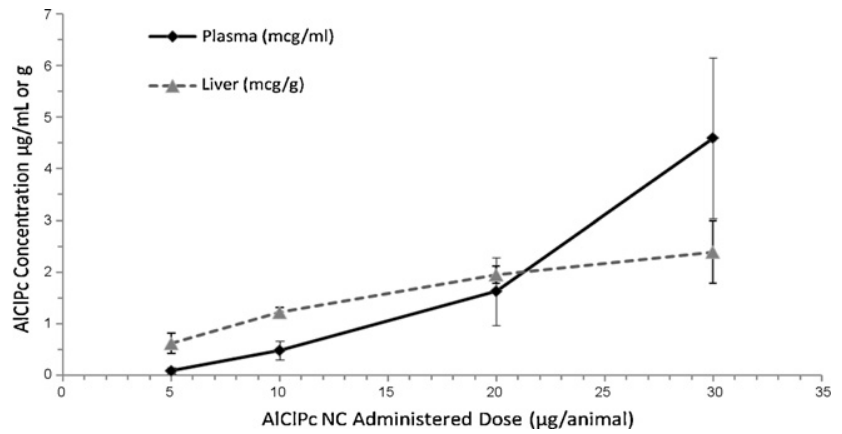

Fig. 3. Concentration of AlClPc in mice plasma and liver 20 min after administration of different doses of AlClPc in PLA formulation $(n=4),{ }^{*} p<0.05$.

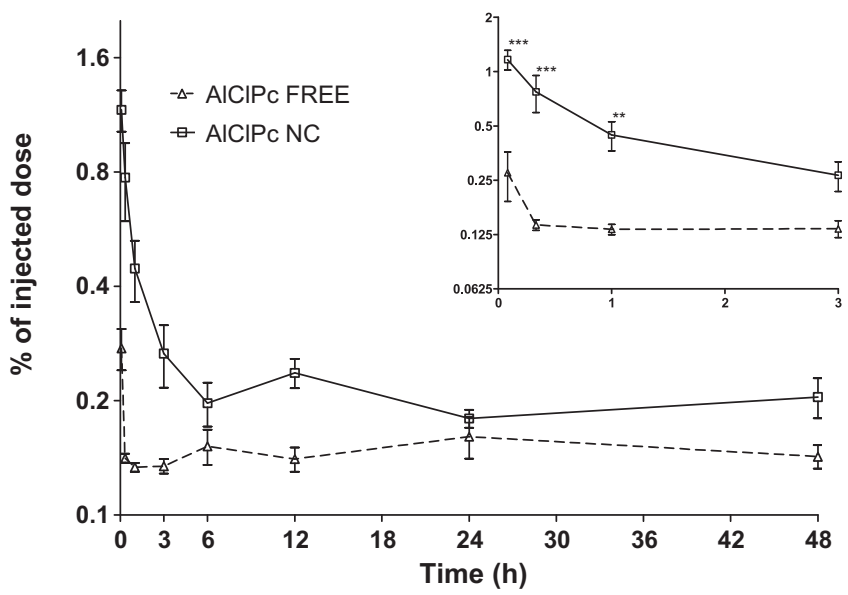

Fig. 4. Plasma AlClPc concentration-time profiles after intravenous administration of $150 \mu \mathrm{l}$ of $0.1 \mathrm{mg} / \mathrm{ml}$ solution of AlClPc free and $0.1 \mathrm{mg} / \mathrm{ml}$ AlCIPc in PLA-NC suspension in mice (mean $\pm \mathrm{SE}, n=6$ ). ${ }^{* * *} p<0.001,{ }^{* *} p<0.01$, using two-way ANOVA.
Table 5

AlCIPc association with different nanocapsule formulations determined by the analytical method.

\begin{tabular}{llll}
\hline $\begin{array}{l}\text { AlClPc } \\
\text { nanocapsules } \\
(0.1 \mathrm{mg} / \mathrm{ml})\end{array}$ & $\begin{array}{l}\text { Encapsulation } \\
\text { efficiency (\%) }\end{array}$ & $\begin{array}{l}\text { Drug } \\
\text { loading }\end{array}$ & $\begin{array}{l}\text { AlClPc }^{\mathrm{b}} \\
\text { precipitated (\%) }^{\mathrm{a}}\end{array}$ \\
\hline PLA & 71.9 & 100 & 9.2 \\
PLA-PEG & 86.4 & 100 & 7.1 \\
PLA-CS & 35.3 & 100 & 17.3 \\
\hline
\end{tabular}

a Drug loading was determined after NC suspension filtration in $0.8 \mu \mathrm{m}$ filter.

b Precipitate recovery after filtration of NC suspension in $0.8 \mu \mathrm{m}$ filter.

indicating that nanocapsules concentrate the dye in the plasma and liver. At the higher doses ( $30 \mu \mathrm{g} / \mathrm{animal})$, a saturation of MPS seems to be reached because the AlClPc concentration in plasma exhibited an exponential increase. The plasma and liver AlClPc concentrations were found to be dose-dependent (Fig. 3).

The pharmacokinetic profiles obtained after intravenous administration of AlClPc solution and AlClPc loaded in PLA-NC are shown in Fig. 4. The plasmatic concentration of AlClPc was significantly higher $(p<0.001)$ than the concentrations of the free AlClPc after 5 20 and 60 min of injection. After $3 \mathrm{~h}$, the plasmatic concentration declined sharply until $6 \mathrm{~h}$. After $3 \mathrm{~h}$ AlClPc concentration of both formulations were similar indicating that the AlClPc was probably released from the NC. It can be noted that nanocapsules modified the plasmatic profile of AlClPc, such as shown in Table 6. The AUC was increased approximately 1.5 times and clearance was reduced by encapsulating this drug in these polymeric nanocarriers.

Table 6

Pharmacokinetic parameters of AlClPc in different formulations after intravenous administration.

\begin{tabular}{lcl}
\hline Parameter & AlClPc free & $\begin{array}{l}\text { AlClPc loaded } \\
\text { in PLA-NC }\end{array}$ \\
\hline AUC $_{0-48}(\mathrm{ng} \mathrm{min} / \mathrm{ml})$ & $429.9^{* * *}$ & 627.8 \\
Clearance $(\mathrm{ml} /(\mathrm{min} \mathrm{kg}))$ & $1237.56^{* * *}$ & 761.46 \\
\hline
\end{tabular}

AUC, area under curve; NC, nanocapsules. Administered doses: $478 \mu \mathrm{g} / \mathrm{kg}$ free AlClPc, $570 \mu \mathrm{g} / \mathrm{kg}$ AlClPc NC.

*** $p<0.001$. 
In the present work, only liver and plasma assays are reported; however, preliminary results indicated that this method could also be used with other tissues, such as brain, lungs, muscle, spleen, kidneys and tumor tissues with the similar LOQ and recovery (data not shown).

\section{Conclusion}

New, specific, accurate, and precise isocratic reverse-phase HPLC methods with fluorescence detection were described for the determination of AlClPc in different polymeric nanocapsule formulations and in biological samples. The method was developed to assess data on the pre-clinical evaluation of AlCIPc as a PDT sensitizing agent. The method was sensitive and robust and could be used in further biodistribution and pharmacokinetic studies and in routine analysis of PDT therapy with this photosensitizer. It is suggested that this method could be useful in AlClPc clinical trials.

\section{Acknowledgements}

The authors thank UFOP for personal financial support to the first author and the CNPq-Brazil researcher grant provided to VCF Mosqueira. This work was also supported by FAPEMIG (APQ-440307 project) and by the NANOBIOMG Network, Minas Gerais, Brazil.

\section{References}

[1] Z.Z. Öztürk, N. kilinç, D. Atilla, A.G. Gürek, V. Ahsen, Recent studies in chem ical sensors based on phthalocyanines, J. Porphir. Phthalocyanines 13 (2009) 1179-1187.

[2] A.P. Castano, T.N. Deminova, M.R. Hamblin, Mechanism in photodynamic therapy: part three. Photosensitizer pharmacokinetics, biodistribution, tumo localization and modes of tumor destruction, Photodiag. Photodyn. Ther. 2 (2005) 91-106.

[3] M. Triesscheijn, P. Baas, J.H. Schellens, F.A. Stewart, Photodynamic therapy in oncology, Oncologist 11 (2006) 1034-1044.

[4] M.M. Zuk, K. Tyczkowska, E. Ben-Hur, H.C. Newman, I. Rosenthal, S.W. Crane, Reversed-phase liquid chromatographic determination of chloroaluminum phthalocyanine tetrasulfonate in canine tissues and fluids, J. Chromatogr. 433 (1988) 367-372.

[5] T.J. Dougherty, C.J. Gomer, B.W. Henderson, G. Jori, D. Kessel, M. Korbelik, J. Moan, Q. Peng, Photodynamic therapy, J. Natl. Cancer Inst. 90 (1998) 889-905.

[6] A. Juarrans, P. Jaén, F. Sanz-Rodriguez, J. Cuevas, S. González, Photodynamic therapy of cancer, basic principles and applications, Clin. Transl. Oncol. 3 (2008) 148-154.

[7] E. Aisling, O‘Connor, W.M. Gallaher, A.T. Byrne, Porphyrin and nonporphyrin photosensitizer in oncology: preclinical and clinical advances in photodynamic therapy, Photochem. Photobiol. 85 (2009) 1053-1074.
[8] Y. Sadzuk, F. Iwasaki, I. Sugiyamaa, K. Horiuchi, T. Hiranod, H. Ozawa, N. Kanayam, N. Okub, Phototoxicity of coproporphyrin as a novel photodynamic therapy was enhanced by liposomalization, Toxicol. Lett. 182 (2008) 110-114.

[9] C.M. Cassidy, M.M. Tunney, P.A. McCarron, R.F. Donnelly, Drug delivery strategies for photodynamic antimicrobial chemotherapy: from benchtop to clinical practice, J. Photochem. Photobiol. B. 95 (2009) 71-80.

[10] K. O‘Riordan, O.E. Akilov, T. Hassan, The potential for photodynamic therapy in the treatment of localized infections, Photodiag. Photodyn. Ther. 2 (2005) 246-262.

[11] P. Klusona, M. Drobeka, A. Kalaji, S. Zarubova, J. Krysa, J. Rakusan, Singlet oxygen photogeneration efficiencies of a series of phthalocyanines in well-defined spectral regions, J. Photochem. Photobiol. A Chem. 199 (2008) 267-273.

[12] S.M.T. Nunes, F.S. Sguilla, A.C. Tedesco, Photophysical studies of zinc phthalocyanine and chloroaluminum phthalocyanine incorporated into liposome in the presence of additives, Braz. J. Med. Biol. Res. 37 (2004) 273-284.

[13] M. Kyriazi, E. Alexandratou, D. Yova, M. Rallis, T. Trebst, Topical photodynamic therapy of murine non-melanona skin carcinomas with chloroaluminum phthalocyanine and a diodo laser: pharmacokinetics, tumor response and cosmetic outcomes, Photodermatol. Photoimmunol. Photomed. 24 (2008) 87-94.

[14] L. Lilge, C. O'Carrol, B.C. Wilson, A solubilization technique for photosensitizer quantification in ex vivo tissue samples, J. Photochem. Photobiol. B. 39 (1997) 229-235.

[15] D.K. Chatterjee, L.S. Fong, Y.Zhang, Nanoparticles in photodynamic therapy: an emerging paradigm, Adv. Drug Deliv. Rev. 60 (2008) 1627-1637.

[16] D. Bechet, P. Couleaud, C. Frochot, M.-L. Viriot, F. Guillemin, M. Barberi-Heyob, Nanoparticles as vehicles for delivery of photodynamic therapy agents, Trends Biotechnol. 26 (2008) 612-621.

[17] M.P. Siqueira-Moura, F.L. Primo, A.P.F. Peti, A.C. Tedesco, Validated spectrophotometric and espectrofluorimetric methods for determination of chloroaluminum phthalocyanine in nanocarriers, Pharmazie 65 (2010) 9-14.

[18] H. Fessi, F. Pusieux, J.P. Devissaguet, N. Ammoury, S. Benita, Nanocapsule formation by interfacial polymer deposition following solvent displacement, Int. J. Pharm. 55 (1989) R1-R4.

[19] V.C.F. Mosqueira, P. Legrand, G. Barrat, Surface-modified and conventional nanocapsules as novel formulations for parenteral delivery of halofantrine, J. Nanosci. Nanotechnol. 6 (2006) 3193-3202.

[20] T. Bohn, T. Walczyk, Determination of chlorophyll in plant samples by liquid chromatography using zinc-phthalocyanine as an internal standard, J. Chromatogr. A. 1024 (2004) 123-128.

[21] ICH Harmonised Tripartite guideline, International Conference on Harmonisation of Technical Requirements for Registration of Pharmaceuticals for Human Use, Validation of Analytical Procedures: Text and methodology Q29(R1), ICH Steering Committee, Geneva, Switzerland, 2005, pp. 1-13.

[22] Committee on Care and Use of Laboratory Animals, Guide for the Care and Use of Laboratory Animals, Natl. Inst. Health, Bethesda, DHHS, 1985, Publ. No. (NIH), pp. 86-123.

[23] US Food and Drug Administration, Guidance for Industry, Bioanalytical Method Validation, Centre for Drug Evaluation and Research (CDER), Rockville, 2001.

[24] C.S. Auletta, Acute subchronic and chronic toxicology, in: M.J. Derelanko, M.A. Hollinger (Eds.), CRC Handbook of Toxicology, CRC Press, Boca Raton, 1995, pp. 51-103.

[25] M.I. Uvarova, G.D. Brykina, O.A. Shpigun, Porphyrins and phthalocyanines in high-performance liquid chromatography. J. Anal Chem 55 (2000) 910-925.

[26] M. Sommerauer, C. Rager, M. Hanack, Separation of 2(3),9(10),16(17),23(24)tetra substituted phthalocyanines with newly developed HPLC phases, J. Am. Chem. Soc. 118 (1996) 10085-10095. 